\title{
Science Opportunities and Capabilities Enabled by LCLS-II and LCLS-II HE
}

\section{X-Ray Science with LCLS-II and LCLS-II HE}

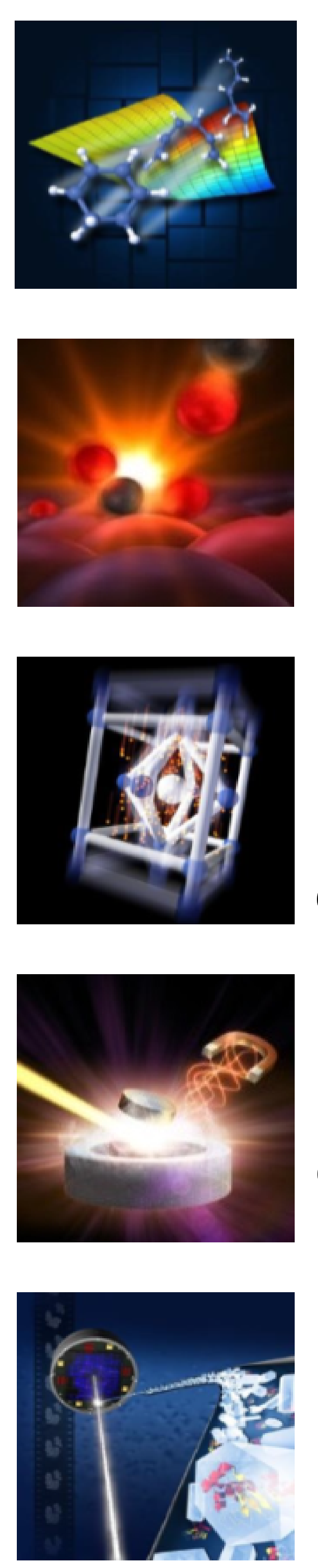

Chemical dynamics:

Reaction dynamics, charge transfer,

molecular photocatalysts, natural $\&$ artificial

photosynthesis

\section{Catalysis:}

Homogeneous and heterogeneous catalysis interfacial \& geo/environmental chemistry

\section{Materials Physics:}

Heterogeneity, spontaneous fluctuations, nonequilibrium dynamics,

extreme environments

\section{Quantum Materials:}

Emergent phenomena \&

collective excitations

Biological Function

\& Structural Dynamics:

Dynamics in physiological environments

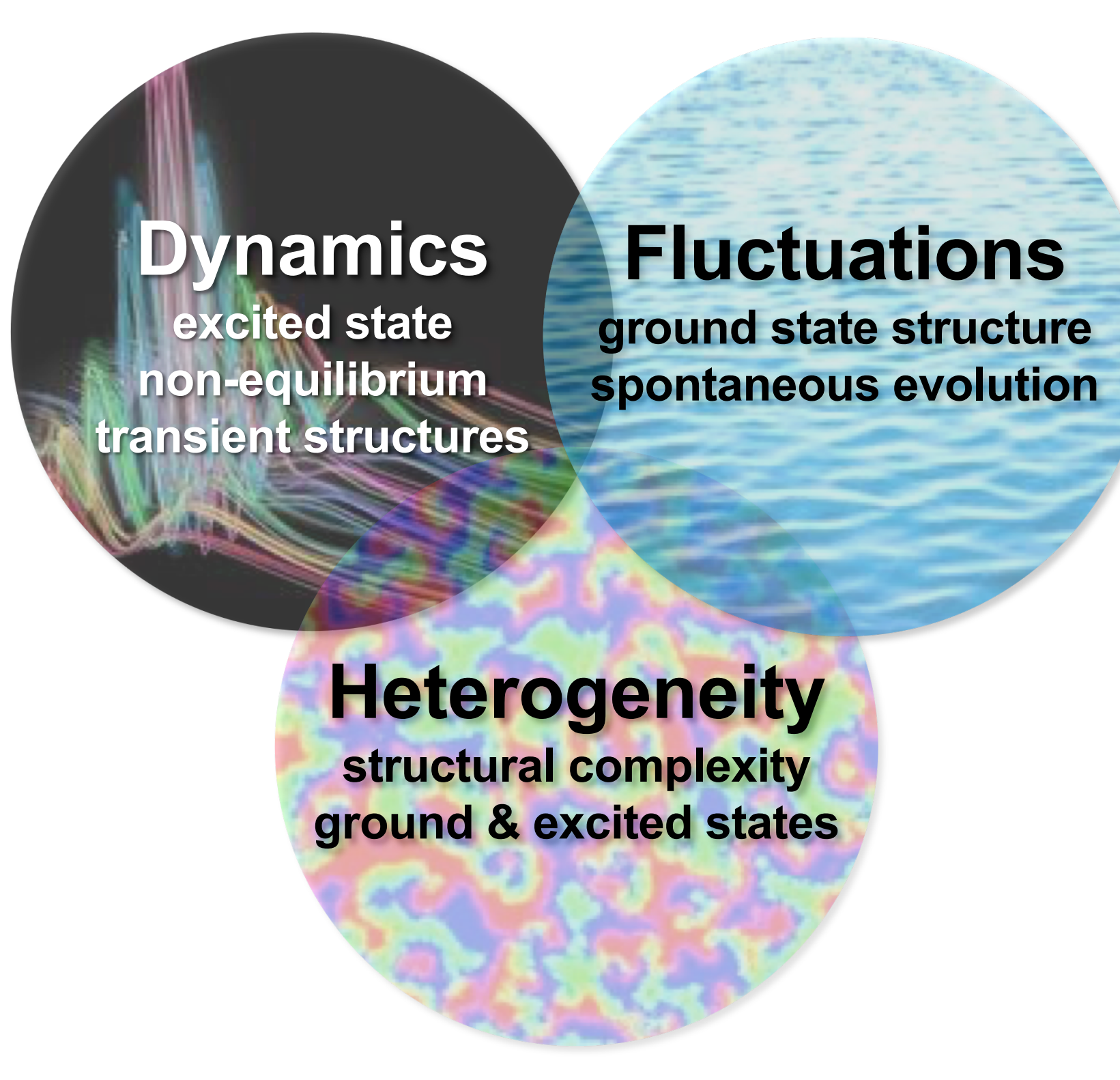

\section{Advancing X-ray Beam Brightness Compared to Previous State-of-the-Art}

LCLS (Linac Coherent Light Source) is an $\mathrm{x}$-ray source that began delivering $x$-rays to scientific experimenters in 2009. Based on a copper linac, it could deliver $x$-rays at a repetition rate of $120 \mathrm{~Hz}$. The LCLS-II linac will use superconducting radiofrequency (SRF) cavities, providing a vast increase in repetition rate, up to $\mathrm{MHz}$. This corresponds to a gain of approximately 3000 in x-ray beam brightness.

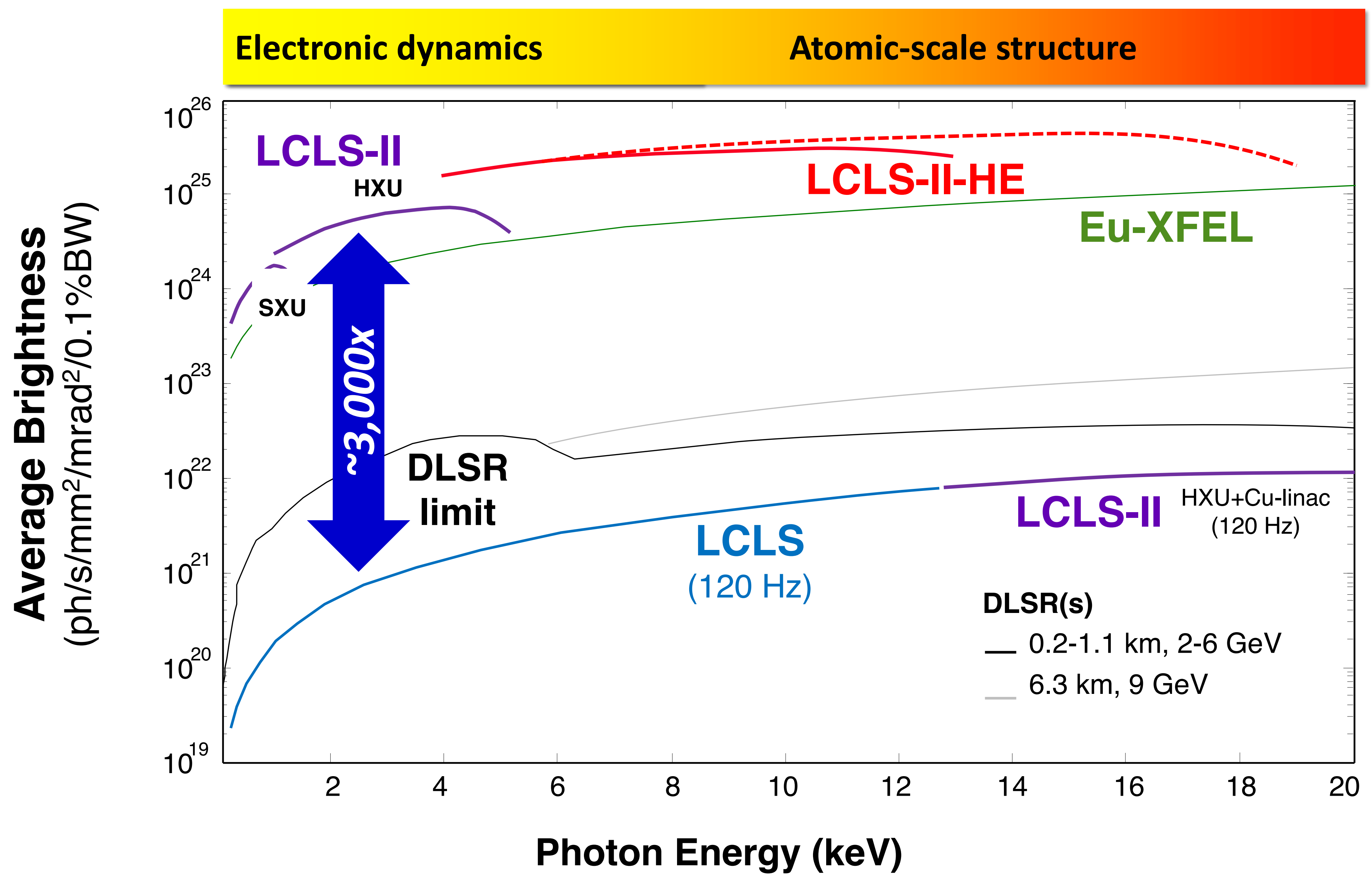

Comparison of LCLS-II and LCLS-II HE x-ray beams to previous state-of-the-art $x$-ray lasers at LCLS (copper linac at SLAC) and Eu-XFEL (superconducting linac at DESY).

\section{High Brightness Beams Make New Science Possible}

A full 3D $\left(1 \mathrm{~cm}^{2} / 10 \mathrm{~nm}\right)$ reconstruction could require up to 100 billion frames

- LCLS today (average flux = storage ring) would take 25 years

- LCLS-II-HE at $100 \mathrm{kHz}$ would take 10 days

- LCLS-II-HE at $1 \mathrm{MHz}$ would take 25 hours

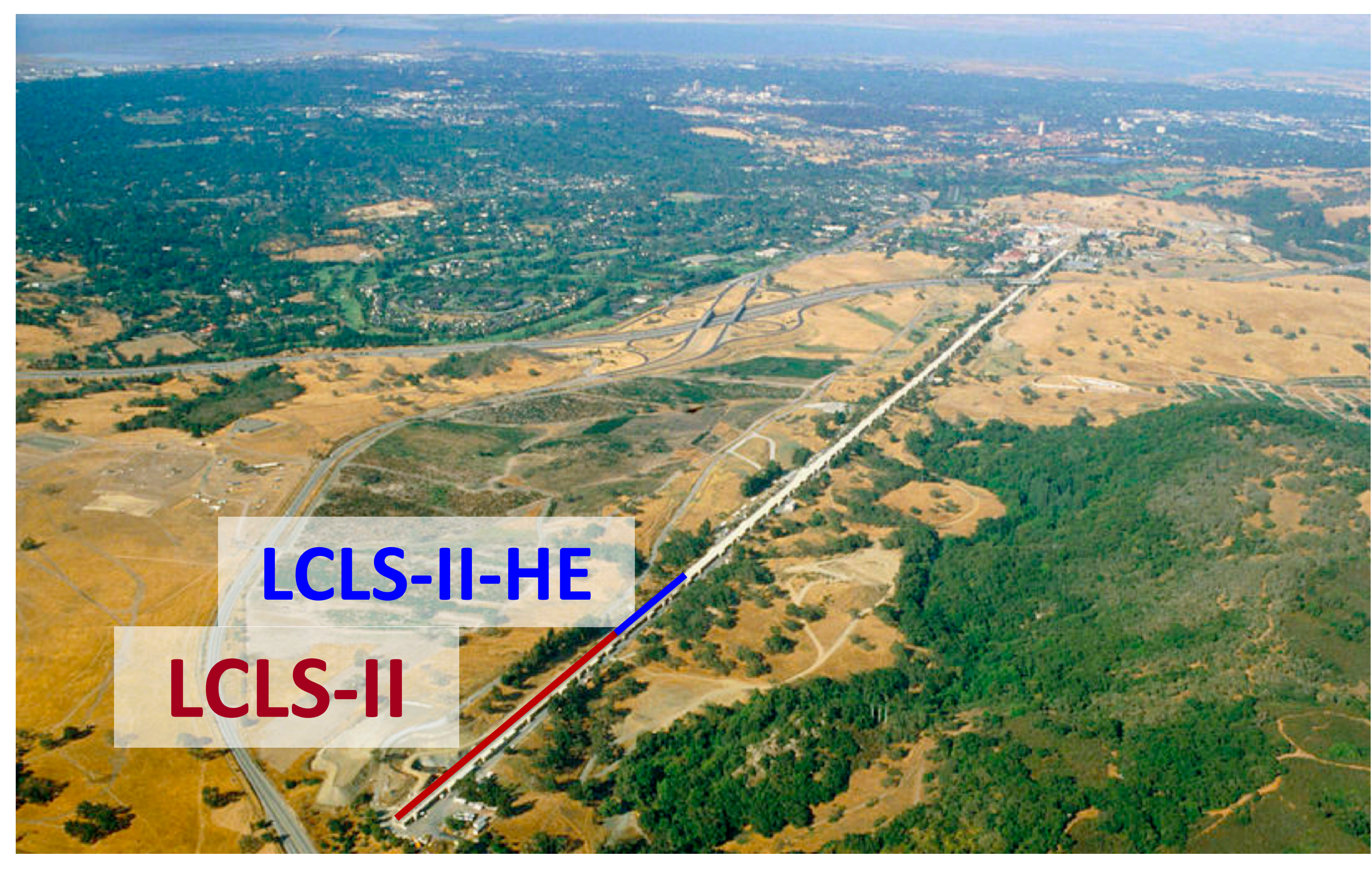

LCLS-II and LCLS-II HE layout on SLAC campus

\section{Fermilab SRF Science Advances that Make LCLS-II and LCLS-II HE Possible}

LCLS-II and LCLS-II HE require superconducting linear accelerators that operate with $100 \%$ duty factor. This creates an enormous load for the cryogenic plant. To make the cryogenic costs manageable, it is crucial to have cavities with extremely high $Q_{0}$ (quality factor). This is made possible by Fermilab SRF science advances from the last 6 years:

- Nitrogen doping: Fermilab researchers developed a technique to add nitrogen impurities to niobium cavities via high temperature heat treatment with $\mathrm{N}_{2}$ gas. This technique is now industrialized and cavity vendors have delivered $>300 \mathrm{~N}$-doped cavities for LCLS-II that reach $Q_{0}$ values 2-3 times higher than was possible with previous techniques.

- Flux expulsion: Fermilab researchers discovered that by giving cavities high temperature heat treatment and cooling them down with a thermal gradient makes it possible to expel magnetic field from the superconductor. This protects the cavities from Q0 degradation. This is also implemented in LCLS-II cavities.
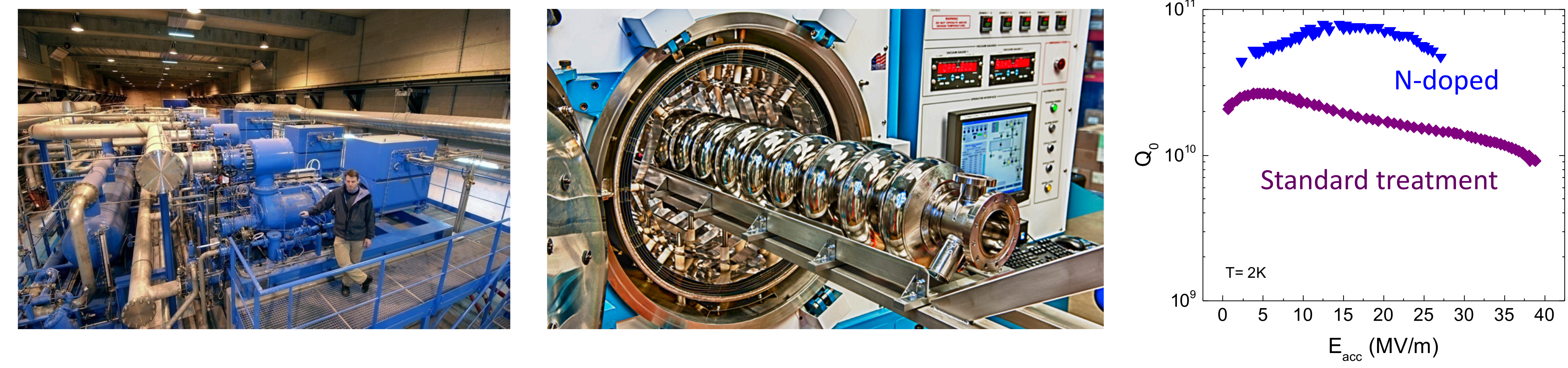

Cryogenic plants (left) for SRF cavity operation require substantial infrastructure and operating costs. Fermilab science involving nitrogen doping (middle) and magnetic flux expulsion enable high quality factors (right) to minimize cryogenic requirements 